\title{
El concepto de vida en El alma del mundo de Schelling*
}

The Concept of Life in Schelling's Von der Weltseele

Violeta Aréchiga ${ }^{\dagger}$

\begin{abstract}
Resumen
La Naturphilosophie de Schelling, parece encajar cómodamente bajo la etiqueta de vitalismo dada la conocida centralidad que juegan, en su sistema, las nociones de organismo y de lo orgánico. Sin embargo, esta caracterización es problemática. Empezando por la existencia de diferentes versiones de lo que significa ser vitalista. El vitalismo, de acuerdo con Lemaitre (2013) "postula la existencia de un principio vital inmaterial considerado como la causa de los fenómenos que tienen que ver específicamente con la vida orgánica". Para Benton (1974), en contraste, "el vitalismo es la creencia de que hay fuerzas, propiedades, poderes o 'principios' que no son físicos ni químicos y que actúan en los seres vivos, o son poseídos por estos, y de que cualquier explicación que no haga referencia a esas propiedades, fuerzas, poderes o principios será incompleta”. Esta última definición no vincula necesariamente al vitalismo con la postulación de un principio inmaterial. Si adoptamos el punto de vista de Lemaitre, tendríamos que la filosofía de Schellling no es vitalista, mientras que si aceptamos la postura de Benton sí lo sería. Más allá de las definiciones, aquí me concentro en analizar la concepción de lo vivo que Schelling. Hago ese examen de la noción de vida de Schelling centrándome en el texto de 1798, Sobre el alma del mundo (Von der Weltseele). De ahí discuto en qué sentido puede ser considerado un pensador vitalista y, asimismo, mostrar de qué manera, en esa obra, se vinculan para él lo físicoquímico y lo específicamente biológico. En segundo lugar, y en relación con la conexión entre lo inerte y lo vivo, el concepto mismo de "alma del mundo" nos conduce a nociones renacentistas y de la modernidad temprana en las que o bien la naturaleza se concebía como un todo vivo o bien persistían ecos de esta concepción. Termino mostrando una parte de la historia de la noción de 'alma del mundo' a fin de entender por qué Schelling hace uso de una idea tan antigua a fines del siglo XVIII; esto permite, desde otra perspectiva, aclarar también qué clase de vitalismo era el schellingniano.
\end{abstract}

Palabras clave: Naturphilosophie - vitalismo - materialismo - organismo - éter

\begin{abstract}
Given the centrality that notions like organism and the organic have in Schelling's Naturphilosophie, it seems in a first instance to respond to a typical definition of vitalism. This interpretation has several problems. Some of these stemming from the different views of how to characterize vitalism. Lemaitre (2013) demands that vitalists postulate an immaterial vital principle as the cause of organic life. Benton (1974) by contrast asserts that vitalism is the belief that there are forces, powers or principles, that are neither physical or chemical, and which affect living organisms or are possessed by them. These second view does not need the immateriality of the cause as a requisite. I argue that if we adopt Lemaitre's view Schelling's philosophy would not be vitalist, whereas under Benton's view it would. Beyond the definitional issue, I engage in this paper in an analysis of Schelling's view of Life centered on his 1798 work Von der Weltseele. I discuss from there to what extent can Schelling be considered a vitalist thinker.
\end{abstract}

Keywords: Naturphilosophie - vitalism - materialism - organism - ether.

\footnotetext{
* Recibido: 30 de Marzo de 2016. Aceptado con revisiones: 5 de Diciembre de 2016.

† Departamento de Humanidades, Universidad Autónoma Metropolitana-Cuajimalpa. Para contactar al autor, por favor, escribir a: violetare@gmail.com.

Metatheoria 8(2)(2018): 157-168. ISSN 1853-2322.

(C) Editorial de la Universidad Nacional de Tres de Febrero. Publicado en la República Argentina.
} 


\title{
1. La noción de vida en El alma del mundo
}

El texto de Schelling titulado El alma del mundo, de 1798, parte de un claro presupuesto: la organización y la vida pueden y deben explicarse mediante principios naturales. Con el fin de elaborar esta explicación, pero asimismo para ofrecernos la concepción de la naturaleza propia de su Naturphilosophie, el autor divide la obra en dos partes, la primera de las cuales trata del mundo inorgánico mientras que la segunda se ocupa del mundo orgánico.

En esta segunda parte lo primero que se nos presenta es un contraste entre la vida animal y la vida vegetal y, como es frecuente en Schelling, dicho contraste involucra dos procesos que se oponen entre sí: la oxidación y la desoxidación en este caso. Para él, la vegetación es una desoxidación continua, el lado negativo de un proceso vital cuyo lado positivo está constituido por la oxidación constante que conforma la vida animal. La idea tras esta propuesta es relativamente sencilla y tiene poco que ver con los procesos que hoy conocemos bajo esos nombres: las plantas expuestas a la luz exhalan aire vital; en contraste, los animales exhalan un "tipo de aire" irrespirable. La planta se desprende del principio vital al paso que el animal lo retiene. En este sentido, encontramos en la base de la vida vegetal y animal dos procesos de orden químico que, nos explica Schelling, no sólo son centrales en los seres vivos sino también en la naturaleza inorgánica. En efecto, en este último terreno, la mayoría de las modificaciones de los cuerpos se relacionan con igualmente con los principios positivo y negativo de la combustión. Sin embargo, esta oposición entre oxidación y desoxidación, entre animal y vegetal, apenas nos ofrece una imagen general de lo que nuestro autor considera que es la vida:

\begin{abstract}
Es bastante evidente que la vida consiste en un constante devenir y que cada producto está muerto precisamente porque es un producto; de donde se sigue el balance de la naturaleza entre dos objetivos opuestos: alcanzar el equilibrio de principios contrarios y mantener sin embargo el dualismo (la única cosa en la que ella misma se perpetúa); es en este balance de la naturaleza (que no deviene jamás producto) que cada ser viviente encuentra su perpetuación (Schelling [1798] 2007, p. 120).
\end{abstract}

Para avanzar en su explicación naturalista de la vida "el concepto de vida debe ser construido, es decir, debe explicarse en tanto que fenómeno natural” (Schelling 2007[1798], p. 122). Schelling considera tres alternativas posibles:

(a) Que el fundamento de la vida resida en la materia misma.

(b) Que el fundamento de la vida resida fuera de dicha materia.

(c) Que el fundamento de la vida resida en dos principios opuestos: un principio positivo fuera del individuo viviente y un principio negativo al interior del individuo mismo.

Pero, para Schelling, la vida no puede ser una propiedad de la materia. Y la razón por la cual se niega a aceptar esta primera alternativa tiene que ver tanto con la teoría de la materia que había expuesto un año antes en Ideas para una filosofía de la naturaleza como con su concepción particular de diferentes órdenes de actividad natural irreductibles entre sí. Cuando dos sustancias entran en contacto entre sí, afirma, se ponen en movimiento recíprocamente; pero ese movimiento no puede explicarse en virtud de las leyes del impacto o colisión. Al contrario, lo que está ocurriendo ahí pertenece a una esfera "más elevada" de operaciones de la naturaleza y es que la materia es en realidad producto de fuerzas opuestas; del equilibrio, perturbado, de la interacción de esas fuerzas. En el terreno de las operaciones químicas estamos justamente ante una alteración del equilibrio primitivo y, por tanto, en este sentido, cada proceso químico es un devenir de nueva materia. Esto significa que los fenómenos químicos no se pueden explicar ni mediante las meras leyes de la colisión ni mediante la ley de la gravedad, es decir, en otras palabras, que la esfera de la química es irreductible a la de la física. Ahora bien, los verdaderos fisiólogos, dice Schelling, coinciden en que las operaciones animales tampoco se pueden explicar mediante las leyes físicas "y a partir de este hecho podemos desde ya suponer una secreta analogía" entre la esfera química y la de la vida" (Schelling [1798] 2007, p. 124). 
Y por supuesto hay más. "La esencia de la organización” se encuentra en que, en esa esfera, la materia y la forma son inseparables; la materia organizada se caracteriza porque aparece individualizada "hasta el infinito". Ya que sólo aparece materia en las operaciones químicas, ya que sólo en éstas podemos concebir la formación hacia una forma determinada, "no nos equivocamos [...] cuando creemos reconocer en las penetraciones químicas el golpe de mano secreto de la naturaleza, del cual se sirve en su individualización constante de la materia" (Schelling [1798] 2007, p. 124).

No obstante, no sería posible afirmar que, para Schelling, la vida sea sin más un proceso químico. En lugar de decir que la vida es un proceso químico, es más "natural", afirma, decir que algunos procesos químicos son procesos imperfectos de organización. Es más razonable suponer que el "impulso formativo" general de la naturaleza muere finalmente en sus producciones inanimadas, que creer, a la inversa, que la tendencia mecánica a la cristalización pueda elevarse hasta dar lugar a formaciones vivientes.

La cuestión es, en el fondo, que aun cuando la formación de la materia viva tenga que explicarse mediante analogías químicas, tenemos que reconocer que esa formación presupone ya la vida misma. Así:

La vida no es una propiedad ni un producto de la materia animal, sino que, a la inversa, la materia es un producto de la vida. El organismo no es una propiedad de objetos naturales particulares, sino que, a la inversa, los objetos naturales particulares son otras tantas limitaciones o modos de intuición particulares del organismo general (Schelling [1798] 2007, p. 125).

En la concepción de Schelling, las cosas no constituyen el principio del organismo, sino al revés: el organismo es el principio de las cosas. "La esencia de todas las cosas [...] es la vida" (Schelling [1798] 2007, p. 126). Por tal motivo, la causa de la vida debe existir con anterioridad a la materia; no debe buscarse en la materia viviente sino fuera de ella.

El siguiente supuesto, que el fundamento de la vida reside por entero fuera de la materia viva, es desechado con mucha rapidez por Schelling. No es posible, para él, concebir un principio positivo al que no se le oponga, de manera necesaria, un principio negativo; en consecuencia, a un principio positivo de la vida, que se hallara fuera de la materia viva, tendría que corresponder un principio negativo al interior de esa materia. La verdad, afirma, tiene que estar en la unión de los dos extremos. Desde este punto de vista, la posibilidad del proceso vital involucra tres presupuestos:

(a) Una causa que mantenga, sin interrupciones, el proceso vital.

(b) Ciertas condiciones negativas, es decir, los principios materiales cuyo conflicto, ya sea separación o reunión, constituye el proceso vital mismo.

(c) Un principio positivo único, en contraste con los principios negativos, que son diversos.

La contradicción más general entre el principio positivo de la vida y sus principios negativos, se reproduce al interior de estos últimos y el antagonismo entre éstos se mantiene en virtud, justamente, de la influencia continua del principio positivo o primera causa de la vida. Como habíamos dicho, para Schelling en la vegetación se produce de manera continua un proceso de desoxidación; se trata de una perturbación del equilibrio efectuada por una causa capaz de lograr que el oxígeno se desprenda y esa causa es la luz. Así, "la influencia de la luz es [...] la primera condición de toda vegetación" (Schelling [1798] 2007, p. 132). En el animal ocurre un proceso inverso: la vida animal es entendida en este marco como un proceso de oxidación -o, lo que es lo mismo para la química de finales del siglo XVIII, de deflogistificación-. Esto supone, a su vez, que el equilibrio de principios en la vida animal tiene que verse perturbado por un aporte continuo de flogisto. De tal manera, los principios negativos de la vida en el cuerpo animal son el flogisto y el oxígeno; el animal descompone el oxígeno mediante la respiración en la misma medida en que elabora flogisto y, ya que el oxígeno es aportado sin cesar al cuerpo, se produce una perturbación constante del equilibrio entre ambos principios. "Es solamente en este perpetuo restablecimiento y esta perpetua perturbación del equilibrio que consiste precisamente la vida” (Schelling [1798] 2007, p. 136). 
Por otro lado, la esencia de la nutrición consiste en un aporte continuo de azote; mediante este aporte, la naturaleza intenta restablecer el equilibrio de los principios negativos de la vida. Al aumentar la cantidad de azote en el cuerpo, la naturaleza tiene como objetivo restablecer el equilibrio dinámico de los principios negativos y esto es posible porque el azote es la sustancia más capaz de fijar el oxígeno. Así, la atmósfera, "el vapor que rodea a la Tierra", contiene los dos elementos cuyo conflicto parece producir la vida.

Si el secreto de la vida reside en un conflicto de principios negativos, uno de los cuales parece luchar contra la vida (el principio azótico), mientras que el otro parece siempre alentar de nuevo la vida [oxígeno], entonces la naturaleza ha puesto ya en la atmósfera el esbozo de la vida general en la Tierra y el hombre [...] comparte su origen etéreo (Schelling [1798] 2007, p. 137).

En este proceso las leyes naturales no dejan de actuar. Para Schelling, la naturaleza no puede sustraer los principios materiales de la vida a las leyes generales que ella misma le ha impuesto a la materia. Esto no significa, sin embargo, que la naturaleza "abandone" totalmente la materia orgánica a la fuerza de atracción. Al contrario, como resultado de las tendencias antagónicas entre una materia inerte, que busca el equilibrio, y una naturaleza animada, que lo odia, la masa resultado de los procesos de crecimiento y nutrición es fijada, por así decirlo, bajo una forma y figura determinadas.

El proceso propiamente químico de la vida nos explica [...] solamente los efectos ciegos e inertes de la naturaleza que se producen tanto en los cuerpos como en los productos muertos, pero no nos explica cómo la naturaleza misma mantiene además de cierto modo su voluntad en estos efectos inertes de fuerzas ciegas en el ser viviente, la cual se revela por la estructura, conforme a un fin, de la materia animal, y que evidentemente no es explicable sino mediante un principio que reside más allá de la esfera del proceso químico (Schelling [1798] 2007, p. 139).

De este modo Schelling subraya tanto el carácter indispensable de las operaciones químicas para la vida, como la irreductibilidad de ésta a la esfera de la química. Si bien las condiciones del proceso vital se encuentran en la materia viva, su causa debe buscarse más allá de esa materia.

Ahora bien, no puede suponerse que esa causa sea una fuerza vital que suprime los efectos de las leyes de la naturaleza en los seres vivientes; aceptar esto sería renunciar a la posibilidad de explicar físicamente la organización. Tampoco puede aceptarse que el origen de la organización está en las fuerzas químicas, porque éstas suprimen la libertad de la naturaleza en la formación y organización. Es necesario, entonces, unir ambas explicaciones y esto se logra acudiendo al concepto de impulso formativo. La noción de impulso formativo es

un concepto sintético que, como todo concepto de este tipo, posee dos factores, uno positivo (el principio de la naturaleza, por el cual la cristalización inerte de la materia animal es perturbada incesantemente) y uno negativo (las fuerzas químicas de la materia animal) (Schelling [1798] 2007, p. 152).

En su grado más bajo, el impulso formativo se manifiesta como un impulso formativo general. La materia como tal posee una disposición a la organización, como se ve en el hecho de que se distingue por la figura y la cohesión. Y justamente porque la fuerza formativa prevalece tanto en la naturaleza orgánica como en la inorgánica, es necesario agregar, para explicar la vida, un principio que eleve a la segunda por encima de la primera. En otras palabras, tenemos que explicar de qué manera una fuerza formativa general se transforma en impulso formativo.

El concepto de impulso formativo incluye la idea de que la formación no se produce sólo de manera ciega, es decir, mediante fuerzas propias de la materia en tanto que tal, sino que a lo necesario que reside en esas fuerzas se añade lo contingente de una influencia externa que, perturbando la fuerza formativa de la materia, la obliga al mismo tiempo a producir una forma determinada (Schelling [1798] 2007, p. 181).

La fuerza formativa se convierte pues en impulso formativo cuando a la primera, que sólo da lugar a un efecto inerte, se le suma algo de contingente, es decir, la influencia perturbadora de un principio externo. Ese algo externo, volviendo al principio positivo de la vida, no puede ser otra fuerza, porque la 
vida no es una fuerza única sino, al contrario, un juego de fuerzas, libre, que es mantenido por una influencia externa. Para la vida, son necesarias fuerzas naturales generales que se ponen en juego. Pero la vida posee también un algo contingente en virtud del cual se mantiene dicho juego, y ese algo, "debe ser un principio particular, es decir, en otras palabras, material" (Schelling [1798] 2007, p. 182). Gracias a este principio, de "cierto modo" la naturaleza orgánica queda sustraída a la esfera de las fuerzas naturales generales y es llevada a una esfera superior, la de la vida. Además, ese principio, causante de la vida, tiene que estar extendido por todas partes aun cuando sólo actúe ahí en donde encuentra una determinada receptividad. Otra de sus características debe ser que sea capaz de individualizarse en seres particulares. No puede ser un constituyente del proceso, pues no está sujeto a ninguna afinidad química, y es por tanto inmutable en todo ser vivo. No suprime las fuerzas inertes de la materia en el ser vivo, pero sí les otorga una dirección que ella no podría haber tomado por sí misma. Sin él, las fuerzas llegarían rápidamente al equilibrio o al reposo. Es, finalmente, un principio que si bien desconocemos, fue supuesto ya por la filosofía antigua como primera fuerza de la naturaleza.

Ahora bien, ya que las diversas funciones de la vida se relacionan todas con modificaciones generales de la naturaleza, debemos buscar el principio común a todas ellas en una misma causa. Lo primero que hay que observar en este sentido es el hecho de que

El flujo más abundante de luz tiene como consecuencia un movimiento general en la naturaleza orgánica, que no se puede atribuir sin embargo a la influencia inmediata de la luz misma [...] sino a un principio que se encuentra difundido universalmente y a partir del cual la luz es quizás simplemente producida (Schelling [1798] 2007, p. 184).

Por otro lado, también hay que notar que aunque nuestra fuente de luz no disminuya, ni ocurra un cambio sobresaliente en el clima o la naturaleza del aire, ciertos años se produce una mala cosecha generalizada. Se trata de "cambios meteorológicos" que tienen efectos en los cuerpos sensibles y que no pueden explicarse mediante las propiedades químicas o higrométricas del aire. Esto nos lleva a tener que admitir que, además de los elementos de la atmósfera que podemos representar químicamente, existe un medio particular en virtud del cual se hacen perceptibles los cambios atmosféricos para los cuerpos vivientes. Ese medio es un principio que no sólo nos permite establecer la continuidad entre el mundo inorgánico y el orgánico, sino que, además, enlaza a todo el conjunto de la naturaleza en una organización universal. Debido a estas características, "reconocemos de nuevo en él ese ser que la más antigua filosofía saludaba, en un presentimiento, como el alma común de la naturaleza, y que algunos físicos de esta época identificaban con el éter" (Schelling [1798] 2007, p. 185) -es decir, el Alma del Mundo, que "impregna cada ser como el aliento común de la naturaleza" (Schelling [1798] 2007, p. 128).

\section{Diferentes tipos de vitalismo}

En este texto, Schelling no explica con demasiado detalle el funcionamiento del éter. En términos generales lo que queda claro es su distinción entre tres esferas distintas -la física, la química y la biológica- y la imposibilidad, para él, de reducir lo químico a lo físico y lo biológico a lo químico. Sin embargo, simultáneamente, identifica la causa primera de la vida, lo que él llama su principio positivo, con un principio material, el éter. La función del éter es mantener sin interrupciones el proceso vital, a su vez constituido por el conflicto entre las condiciones negativas de la vida, que en su base son también principios materiales: el oxígeno, el azote y el flogisto.

Si adoptamos una definición común de vitalismo, según la cual éste consiste en postular "la existencia de un principio vital inmaterial considerado como la causa de los fenómenos concernientes específicamente a la vida orgánica" (Lemaitre 2013, p. 7). lo que se puede afirmar de entrada es que la de Schelling no es, al menos en el Alma del mundo, una postura vitalista. Pero, asimismo, numerosos autores coinciden en señalar que el vitalismo presenta diferentes caras e incluso orientaciones hacia el 
materialismo. Quizás en este sentido sea más útil retomar, para analizar la postura de Schelling, un texto de Benton de 1974, en el que este autor nos ofrece otro punto de vista.

De acuerdo con Benton sería posible ofrecer una definición preliminar del vitalismo en los siguientes términos:

Vitalismo es la creencia en que fuerzas, propiedades, poderes o "principios" que no son ni físicos ni químicos actúan en, o son poseídos por, los organismos vivos y en que cualquier explicación de las características distintivas de los organismos vivos que no haga referencia a esas propiedades, fuerzas, poderes o principios será incompleta (Benton 1974, p. 18).

Esta definición, reconoce su autor, origina de manera inmediata una serie de preguntas, entre ellas:

(a) Qué distingue a una fuerza física o química de una que no es tal.

(b) Cuál es el estatus epistemológico de los principios vitales.

(c) Cuál es el estatus ontológico de esos principios.

Benton sostiene que las respuestas a estas preguntas son casi tan numerosas como los vitalistas mismos y que, por lo tanto, lo que se necesita no es una definición de vitalismo sino una tipología. Esta tipología evaluaría una teoría vitalista determinada en tres ejes: su grado de escepticismo epistemológico u osadía ontológica; el tipo de explicación que ofrece y el tipo de problemas que su autor investiga.

En cuanto al grado de escepticismo epistemológico, el eje iría desde una postura fenomenalista, afín al positivismo, en la que los poderes vitales se entienden solamente como relaciones generales a las que se llega mediante la observación de los fenómenos, hasta una postura realista. Al interior de la postura realista, se presentarían diferentes variedades, según el principio vital se considere como una agencia incorpórea, constituyentes materiales específicos o fuerzas o poderes. Las posturas vitalistas también podrían distinguirse en función del tipo de explicación que proponen: teleológico, es decir, explicación mediante causas finales; nomológico, explicación mediante leyes deterministas; y el nonomológico, explicación sin leyes. Finalmente, tendríamos el eje que analiza la postura vitalista en términos de los problemas con los cuales su autor lidiaba y este distinguiría problemas morfogenéticos (el crecimiento, la diferenciación, el desarrollo, la regeneración, entre otros); problemas fisiológicos (mantenimiento de la organización interna en la interacción con el medio, mantenimiento de los compuestos orgánicos, el calor animal); y problemas relacionados con la química (la autonomía, o no, de la química orgánica frente a la inorgánica, por ejemplo).

En lo que se refiere al primer eje, claramente Schelling adopta una postura realista -de osadía ontológica, en palabras de Benton. Esto no se debe solamente a que los principios de la vida que postula, tanto los negativos (oxígeno, flogisto, azote) como el positivo (éter), sean constituyentes materiales específicos, ni a que nuestro autor esté postulando fuerzas o poderes propiamente vitales. Estos constituyentes materiales son únicamente uno de los dos aspectos en la concepción de la vida que estamos analizando. El otro aspecto, esencial, es el del juego de fuerzas, la interacción entre el principio positivo y las condiciones negativas, y la interacción de fuerzas también al interior de las condiciones negativas, entre el oxígeno y el flogisto, o entre el oxígeno y el azote, por ejemplo. Así, Schelling afirma:

Lo propio de la posibilidad de la vida es [...] una sucesión constante de procesos de descomposición y de recomposición, en los que la materia animal no obedece siempre solamente a las leyes ciegas de la afinidad química, sino también a la influencia de la causa positiva de la vida, que no la deja llegar en el cuerpo viviente a una total disolución (Schelling [1798] 2007, p. 161).

El proceso vital es, para Schelling, algo más que la mera suma de sus constituyentes y ese algo más es una tendencia al equilibrio continuamente perturbada y, en consecuencia, mantenida sin interrupción, por sus factores.

En cuanto a la segunda dimensión de Benton, la que tiene que ver con las formas de explicación, podemos ver que hay en la concepción de Schelling tanto una explicación mediante leyes, como una explicación de acuerdo con causas finales. Por un lado, subraya la necesidad no sólo de explicar la vida 
mediante principios naturales, sino de advertir que los principios materiales de la vida no se pueden sustraer a las leyes generales de la materia. En este sentido, estaría defendiendo una explicación de acuerdo con leyes deterministas. Pero, por otro lado, también aborda otro aspecto del proceso vital, que tiene que ver justamente, como mencionaba Benton, con procesos morfogenéticos. En la aparición de materia animal, en otras palabras, en los procesos de nutrición y crecimiento, la materia orgánica no está únicamente a merced de las fuerzas de atracción, sostiene Schelling. Hay aquí también un juego de fuerzas, esta vez entre una tendencia de la materia inerte al equilibrio y una naturaleza animada que lo odia, y en ese juego la "masa muerta" queda detenida bajo una forma y figura determinadas. Ahora bien, aunque la aparición de materia animal en general, se produce solamente en virtud de leyes ciegas y necesarias, la adopción por parte de esa materia de una forma determinada, es un resultado de otro tipo.

El proceso propiamente químico de la vida nos explica [...] solamente los efectos ciegos e inertes de la naturaleza que se producen tanto en los cuerpos como en los productos muertos, pero no nos explica cómo la naturaleza misma mantiene además de cierto modo su voluntad en estos efectos inertes de fuerzas ciegas en el ser viviente, la cual se revela por la estructura, conforme a un fin, de la materia animal, y que evidentemente no es explicable sino mediante un principio que reside más allá de la esfera del proceso químico (Schelling [1798] 2007, p. 139).

La estructura y la vida no se pueden explicar entonces solamente mediante leyes físicas o químicas; se requiere, también aquí, de un principio irreductible y que parece actuar de acuerdo a fines. Es en este contexto que Schelling introduce el concepto de impulso formativo, en el que, afirma, se reúnen la libertad de la naturaleza en la formación y la organización, y su actuar conforme a leyes. Pero como este impulso formativo presupone la existencia de una materia orgánica, es necesario apelar, para explicar la vida, a una causa fuera de la materia organizada misma. Se trata de un principio externo que no está sometido al proceso químico, porque, por sí solas, las leyes químicas conducen únicamente a la detención del proceso natural. Al contrario, si suponemos ese principio

podemos explicar perfectamente [...], en primer lugar, la conformidad ciega a las leyes de la naturaleza en todas sus formaciones, por las fuerzas químicas que en ella concurren, pero también la libertad de esas formaciones, o la contingencia que hay en ellas, por la perturbación, accidental con respecto al proceso químico mismo, de las fuerzas formativas específicas de la sustancia animal por un principio externo, independiente del proceso químico mismo (Schelling [1798] 2007, p. 152).

Finalmente, el tercer eje de Benton, es el de los tres el que resulta más difícil de encajar con Schelling. En efecto, este último autor, aunque continuamente al pendiente de los resultados científicos de su época, es filósofo ante todo. Los problemas de los que se ocupa en El alma del mundo son fisiológicos principalmente, pero toca también, como vimos, cuestiones morfogenéticas y químicas. Sin embargo, sí había un problema filosófico general al que Schelling se había enfrentado desde su texto Ideas para una filosofía de la naturaleza: tratar de mostrar, en contra de Kant, que la realidad en su conjunto es cognoscible en la medida en que existe una identidad entre el sistema del espíritu y el sistema de la naturaleza. Para mostrar esto es que Schelling acude al modelo de lo orgánico: el organismo es un análogo del espíritu en la medida en que posee actividad espontánea, una fuerza interna o juego de fuerzas mediante el cual se produce a sí mismo y una configuración con arreglo a fines. El establecimiento de la identidad entre espíritu y naturaleza involucra así la necesidad de elaborar una concepción determinada de la vida, en la que los objetos naturales particulares son sólo "modos de intuición particulares" del organismo general. Desde este punto de vista, las cosas no pueden ser principios del organismo; al contrario, el organismo general es el principio de las cosas y la esencia de todas estas es la vida (véase Schelling [1798] 2007, pp. 125-126)

En resumen, encontramos que la postura de Schelling se caracteriza por ser realista en la medida en que invoca una serie de fuerzas y principios que, en su interacción y manifestación, son irreductibles a las leyes de la física y de la química. Por otro lado, hace uso en sus explicaciones tanto de leyes deterministas, "ciegas", como de causas finales. Finalmente, aunque Schelling retomaba los resultados de las ciencias de su época, los problemas que le ocupan y que su concepción de la vida pretende 
enfrentar, son de orden filosófico, epistemológico en primera instancia. Si esto es una postura vitalista, sin duda se apoya de manera aparentemente paradójica en varios principios materiales, el oxígeno, el flogisto, el azote y el éter entre ellos. Pero, insiste Schelling repetidamente, la vida no es solamente un proceso químico, porque en este tipo de procesos hay siempre una tendencia al equilibrio. Se requiere la intervención de una causa que altere continuamente ese equilibrio, y esa causa es el principio positivo de la vida, es decir, el éter. El éter no suprime las fuerzas inertes de la materia en el cuerpo viviente, pero, sin él, esas fuerzas llegarían al reposo, es decir, darían lugar sólo a materia muerta.

\section{El éter}

Ahora bien, la historia de las diversas concepciones del éter es todavía más compleja que la de los vitalismos y más extendida en el tiempo. Pero Schelling mismo reconoce que su principio positivo de la vida es el mismo que aquél en que los antiguos habían "supuesto la primera fuerza de la naturaleza" (Schelling [1798] 2007, p. 184), si bien bajo la forma de representaciones poéticas. Luego de toda una revolución, una vuelta completa, afirma, la filosofía está retornando progresivamente a la filosofía antigua.

En la primera parte de El alma del mundo, la que trata de la naturaleza inorgánica, Schelling se refiere también, como hizo al final de su texto, a la atmósfera.

El conocimiento más íntimo de nuestra atmósfera nos dará la clave de una teoría completamente nueva de la naturaleza. La circulación general en la que la naturaleza se perpetúa pasa por la atmósfera; es en ella, como en un taller secreto, que se prepara lo que la primavera tiene de encantador, lo que el verano tiene de terrible; es en ella, en fin, que el naturalista entusiasta ve desde ya la primera disposición, y de cierta manera el esquematismo, de toda la organización sobre la Tierra (Schelling [1798] 2007, p. 90).

A lo que nuestro autor se está refiriendo aquí es al hecho de que de la vegetación se desprende continuamente una gran cantidad de "aire vital". Pero ya que los animales y el fuego consumen cada estación tanto aire puro como el que se ha desprendido de la vegetación "en primavera y verano", resulta verosímil suponer ese aire vital sea exhalado por el sol junto con la luz. El sol sería la causa que cotidianamente "rejuvenece" a la atmósfera y le devuelve lo que había perdido a causa de diversos procesos químicos.

Así pues, dice Schelling, es posible que la luz no sea la única materia que el sol exhala. Tenemos que considerar que todas las materias expansivas, la luz entre ellas, siguen sus propias fuerzas de expansión. De este modo, el espacio vacío al interior de cada sistema solar debe estar lleno de materias con diferentes grados de elasticidad. En esas regiones se encuentra la fuente inexhaustible de fuerzas positivas que se extienden en todas direcciones y que mantienen el movimiento y la vida en los diferentes cuerpos celestes.

Cada cuerpo celeste particular se puede apropiar de estas materias; las reúne en torno a sí bajo la forma de una atmósfera que deviene de ahí en adelante para él la fuente inmediata de todas las fuerzas vivificantes, aun cuando éstas no fluyen hacia él sino a partir de una fuente que reside en regiones muy alejadas.

Regresando al oxígeno de nuestra atmósfera, Schelling lo define como la sustancia elemental ponderable del aire vital. Es, pues, sólo una parte del aire vital, su materia negativa; durante el proceso de combustión, esta materia negativa se liga a los cuerpos, mientras que la materia positiva de ese mismo aire se desprende en forma de luz. Y concluye,

Si, entonces, el aire vital es la fuente de la luz, y el - 0 [el oxígeno] la materia ponderable que limita en sus movimientos y enlaza a los cuerpos en gravitación un fluido que circula libremente, disperso en torno a los cuerpos celestes, extremadamente elástico, entonces la antigua teoría, recientemente exhumada por Descartes, Huygens y Euler, de un éter universalmente extendido, deja de ser, al menos en parte, hipotética, y eso que Newton mismo no osaba sino suponer al final de su Óptica devendrá, quizás, evidente (Schelling [1798] 2007, p. 21). 
Como vemos, los conceptos de aire vital, oxígeno, luz y éter se enlazan aquí de manera particularmente intrincada. A ello se le añade que, en el título de su obra, Schelling está identificando al éter con el alma del mundo. Y es de esta identificación de la que queremos hablar a continuación.

Como Hodge y Cantor explican en su texto de 1981 (véase Cantor \& Hodge 1981). para la época de Newton se le atribuían al éter diversas características, entre ellas el ejercer ciertas fuerzas, estar presente en espacios vacíos de sólidos, fluidos y gases ordinarios, ser imperceptible, y transmitir efectos semejantes o idénticos a los del magnetismo, la electricidad, el calor y los impulsos nerviosos. El éter podía concebirse como material o como inmaterial, como fluido o sólido, continuo o compuesto por partículas, conforme a las leyes de la mecánica o fuera del alcance de éstas. Pero ya para entonces el éter tenía una larga historia; por ejemplo, en Aristóteles el éter del que estaban compuestos los cuerpos celestes poseía un análogo en la Tierra, el pneuma, que, aunque no era un alma como tal, sí era el instrumento de animación en los seres vivos. Luego, con los estoicos, el éter y el pneuma se identificaron.

\footnotetext{
El principio activo, la Naturaleza o Dios, corporizado como pneuma, una mezcla de fuego y aire, algunas veces identificada con el aither, se mezcla por todas partes de manera activa con la materia, penetrándola y dándole forma para constituir cuerpos que pueden ellos mismos actuar y ser objeto de acciones. Este principio activo, como Dios o Naturaleza, es un logos inmanente, una inteligencia racional, providencial, la verdadera vida del universo mismo a partir de la cual toda vida vegetal, animal y humana obtiene su animación y facultades diversas (Cantor \& Hodge 1981, p. 6).
}

Los neoplatónicos, por su parte, parecen haber identificado ese éter-pneuma con el Alma del Mundo, como una entidad capaz de activar los cuerpos, de animarlos, en directo contraste con el carácter inerte de la materia sola. Ya en el Renacimiento, encontramos una filosofía química paracelsiana en la que, dicen los autores, "existen fuertes ecos del pneuma material estoico y de la inmaterial anima mundi neoplatónica" (Cantor \& Hodge 1981, p. 6). Y el tema continúa, pasando por la materia sutil cartesiana hasta llegar a los éteres de Newton y las concepciones de Boerhaave entre muchas otras.

Cantor y Hodge hacen una advertencia a los historiadores en este marco. Resulta sencillo, desde su punto de vista, intentar derivar por ejemplo alguno de los éteres de Newton de los espíritus paracelsianos, en la medida en que ambos cumplen con papeles explicativos parecidos y poseen efectos generales semejantes. Estamos ante una familia explicativa que en efecto incluye al éter de Descartes, el anima mundi de los neoplatónicos, el pneuma de los estoicos, etc. Pero el parecido puede ser superficial; el historiador, nos dicen, debe concentrarse en las grandes diferencias que existen entre todas esas corrientes en lo concerniente a temas como teoría de la materia, alma, sustancia, causación, providencia y leyes naturales.

No obstante, en el caso de Schelling, quizás sea posible vincular su concepción del éter con otra teoría que se difundió durante el Renacimiento, la teoría del espíritu aéreo vital -y ello en la medida en que es él mismo quien, por un lado, identifica el éter con el alma del mundo y, por otro, insiste en la importancia del retorno, que según él se está viviendo en su época, a las "creencias naturales más antiguas y más sagradas del mundo" (Schelling [1798] 2007, p. 65).

Es posible que el que comenzó a pensar primero en términos de lo que después sería llamado "espíritu aéreo vital" haya sido Paracelso. En su "Sobre la naturaleza de las cosas", sostiene que nadie puede negar que el aire le proporciona vida a todas las cosas, cuerpos y sustancias que se producen y generan sobre esta Tierra. ${ }^{1} \mathrm{Al}$ parecer, en otro de sus textos Paracelso mencionó asimismo la semejanza entre la vida y la combustión, indicando que el aire era esencial para ambas. Pero con el tiempo los paracelsianos argumentarían específicamente que sólo cierta parte del aire era el ingrediente esencial en este sentido: el nitro o salitre aéreo; éste a su vez llegó a ser prácticamente identificado con el pneuma de los estoicos y se consideró necesario para todo tipo de vida, ya fuera mineral, animal o vegetal. Esta idea se expresó en ocasiones diciendo que en el aire se encontraba el alimento de la vida (véase Aréchiga 2007).

${ }^{1}$ Véase Paracelso, "Of the Nature of Things", en Linden 2003, p. 155. 
Así, en su Tratado sobre el azufre, publicado en 1604, Michael Sendivogius sostiene que los espíritus vitales de los animales se crean a partir de la "más pura sustancia" del aire, y que éste es un elemento notablemente valioso porque constituye el verdadero sitio de la semilla de todas las cosas. El aire "contiene el espíritu vital de todas las creaturas, espíritu que vive en todas partes, penetra todas las cosas y proporciona su semilla a los otros elementos" (Sendivogius 1722, p. 163). Tanto el hombre como el resto de los animales, dice Sendivogius, mueren si carecen de aire, y nada crecería en el mundo sin la "virtud del aire, que penetra, altera y atrae hacia sí el alimento multiplicador (Sendivogius 1722, p. 164). El aire que nos rodea verdaderamente está repleto de virtud divina, pues en él está incluido el Espíritu del Señor. El alimento secreto de la vida fue identificado por este autor con el nitro o salitre, argumentando entre otras cosas que ésta era la sustancia gracias a la cual todo crecía y se alimentaba, y que la lluvia tenía un poder fertilizante porque recibía un poder vital del aire y lo unía al salitre de la tierra.

En el último cuarto del siglo XVII apareció nuevamente esta idea, esta vez al interior de una visión mecanicista por lo menos a primera vista. ${ }^{2}$ John Mayow retomó la idea de que había algo en el aire que era esencial tanto para la combustión como para la respiración, pero en su caso no era el aire como un todo el que jugaba el papel vital sino sólo una parte de él, considerada de carácter nitroso. "El aire que nos rodea", afirma este autor, "está impregnado de una sal universal de naturaleza nitro-salina, es decir, un espíritu vital, ígneo, y altamente fermentativo" (Mayow [1674] 1907, p. 1). Ahora bien, solamente la parte más volátil y sutil del nitro se deriva del aire; la otra parte se deriva de la tierra. Por este motivo, que "la tierra está impregnada con cierta semilla universal, que fecunda todas las cosas, ha sido por largo tiempo una opinión aceptada" (Mayow [1674] 1907, p. 5). Se trata, dice, de una semilla "macrocósmica" que es, o bien una sal fija, o la semilla de sales fijas ocultas en el seno de la tierra. Cuando estas semillas con el curso del tiempo llegan a su madurez se transforman en la sal nitro, junto con el espíritu nitro-aéreo. Este espíritu, por su parte, cuando se encuentra en un estado de subdivisión muy fina, reside en el aire, permeando todas las cosas gracias a su naturaleza extremadamente penetrante. Es indispensable en la producción de la flama y por esta razón es llamado por Mayow "alimento ígneo-aéreo" (Mayow [1674] 1907, p. 9). Finalmente, es importante notar que este autor declara que

Entre los elementos de las cosas naturales, el espíritu nitro-aéreo tiene el primer sitio, de modo tal que puede con justicia ser llamado Mercurio, ya que es una sustancia excesivamente sutil, ágil y etérea, y es asimismo el instrumento primario de la vida y el movimiento no sólo en las plantas sino también en los animales (Mayow [1674] 1907, p. 34).

Una de las características que une a estas tres visiones renacentistas y de la modernidad temprana es el que sitúan en el aire, en nuestra atmósfera, un principio vital. Este principio es llamado espíritu, tanto en el caso de Sendivogius como en el de Mayow, pero en ambos casos se trata de un espíritu, paradójicamente, material: la "sustancia más pura del aire" o partículas nitro-aéreas. Además, se trata de una entidad que se encuentra difundida universalmente y que se considera muy penetrante, a la cual Mayow específicamente califica de etérea. Es importante notar aquí la función que juegan el aire paracelsiano, el espíritu aéreo vital y el espíritu nitro-aéreo. El hecho de localizar en el aire, o en una parte de él, el principio vital, nos habla de una concepción de la naturaleza en la que ésta, luego de ser creada, es capaz por sí misma de producir importantes efectos, entre ellos nada menos que el de causar la vida. De acuerdo con Sendivogius,

La naturaleza es sólo una, verdadera, clara, perfecta y entera en su propio ser, el cual Dios creó desde el comienzo, poniendo en ella su espíritu [...]. Todas las cosas proceden de esta misma naturaleza sola; y tampoco hay nada en el mundo sin la naturaleza [...]. La naturaleza no es visible aunque actúa visiblemente; porque es un espíritu volátil el que lleva a cabo su oficio en los cuerpos y está situado y sentado en la voluntad y la mente de Dios (Sendivogius, 1722, p. 176).

\footnotetext{
${ }^{2}$ El lenguaje usado por Mayow al aclarar, por ejemplo, que la reacción entre sales fijas y sales ácidas no debe considerarse como una enemistad, sino como un afecto conyugal, permite por lo menos poner en un contexto más amplio su lenguaje, en otros sitios, en términos de partículas.
} 
Es esta concepción de la naturaleza y su actividad la que nos permite establecer una vinculación entre el éter de Schelling y el espíritu aéreo vital.

\begin{abstract}
Baader y Schelling se esforzaron por demostrar [...] la presencia y cooperación activa, en cada evento y sustancia físicos, de un alma del mundo imperceptible, identificada con el éter, material pero no obstante semi-divina o cuasi-divina a causa de su omnipresencia, su capacidad de penetrarlo todo y su efecto vitalizante (Vassányi 2011).
\end{abstract}

\title{
4. Conclusión
}

Es conocida la influencia que tuvo la filosofía químico-alquímica sobre el punto de vista de la Naturphilosophie. ${ }^{3}$ La importancia que esa filosofía tenía para autores como Schelling residía, entre otras cosas, en que el concepto renacentista de la naturaleza permitía construir una visión inmanente de la actividad natural al interior de la cual se podía desarrollar una teleología naturalizada. Desde este enfoque, la naturaleza es activa por sí misma y es capaz de establecer leyes que dan cuenta del modo en que los seres vivos existen y se desarrollan. La Naturphilosophie de Schelling es pues heredera de una tradición que considera que en la naturaleza misma se encuentra un principio inmanente de automovimiento y auto-organización, cuya fuente se encuentra en poderes que residen en la materia y no fuera de ella.

El vitalismo de Schelling resulta ser en todo caso un vitalismo materialista, inmanentista, en el que la interacción natural entre principios materiales y fuerzas constituye lo que da origen a la vida. Sin embargo, el principio positivo que aquí entra en juego, el éter, no es algo que pertenezca exclusivamente a la esfera de lo orgánico. Es, al contrario, un principio común que, afirma Schelling, fluctúa entre la naturaleza inorgánica y la orgánica, y que "contiene la causa de todos los cambios de la primera y la razón última de toda la actividad en la otra" (Schelling [1798] 2007, p. 7). En este marco, nuestro autor no niega que existe una relación esencial entre los procesos químicos y la vida, pero insiste en la irreductibilidad de esta última a los primeros. Lo que se encuentra tras esta postura es una concepción de la naturaleza en su conjunto como un "organismo general", la idea de que "la esencia de todas las cosas [...] es la vida” (Schelling [1798] 2007, p. 126). En este sentido, los procesos químicos son solamente procesos de organización imperfectos, un impulso formativo que muere en producciones inanimadas. La formación de la materia animal, aunque sólo pueda explicarse mediante analogías químicas, presupone ya la vida misma.

Esta concepción de la naturaleza en términos de "organismo general" también acerca a Schelling a una visión renacentista de acuerdo con la cual la naturaleza es entendida, por analogía con un ser vivo, como una agencia creativa y dinámica.

Bibliografía

Aréchiga, V. (2007), Fuego y vida: Fuentes del pensamiento quimico de Buffon, México: CEFPSVLT.

Benton, E. (1974), "Vitalism in Nineteenth-Century Scientific Thought: A Typology and Reassessment", Studies in the History and Philosophy of Science 5: 17-48.

Cantor, G.N. y M.J.S. Hodge (1981), "Major Themes in the Development of Ether Theories from the Ancients to 1900", en Cantor, G.N. y M.J.S. Hodge (eds.), Conceptions of Ether, Cambridge: Cambridge University Press, pp. 1. 60.

\footnotetext{
${ }^{3}$ Véase por ejemplo Vassányi (2011, p. 143). También: "Böhme y Ötinger fueron mediadores no sólo de la teosofía cabalística sino también de la tradición hermético-alquímica para Baader y Schelling, que recurrieron al concepto de un alma del mundo" (Vassányi 2011, p. 394).
} 
168 | Violeta Aréchiga

Lemaitre, J.-C. (2013), “Le statut de l'organisme dans la philosophie schellingienne de la nature”, Klesis-reuve philosophique $25: 3-36$.

Mayow, J. ([1674] 1907), Medico-Physical Works, Londres-Edimburgo: The Alembic Club.

Paracelso (2003), "Of the Nature of Things", en Linden, S.J. (ed.), The Alchemy Reader, Cambridge: Cambridge University Press, pp. 151-169.

Schelling, F. ([1798] 2007), El alma del mundo, París: Éditions Rue D’Ulm.

Sendivogius, M. (1722), A Philosophical Account of Nature in General, Londres: John Hooke.

Vassányi, M. (2011), Anima Mundi: The Rise of the World Soul Theory in Modern German Philosophy, Dordrecht: Springer. 